\title{
JSWSC: recent developments and further advances
}

\author{
Jean Lilensten $^{1, *}$, Anna Belehaki ${ }^{2}$, Jürgen Watermann ${ }^{3}$, Jan Janssens ${ }^{4}$, and Agnès Henri ${ }^{5}$ \\ ${ }^{1}$ Editor-in-Chief, Institut de Planétologie et d' Astrophysique de Grenoble, UGA/CNRS-INSU, Grenoble, France \\ 2 Editor-in-Chief, Institute of Astronomy, Astrophysics, Space Applications and Remote Sensing, National Observatory of Athens, \\ Metaxa and Vas. Pavlou, 15236 Palaia Penteli, Greece \\ ${ }^{3}$ Editorial Advisor, jfwConsult, 83400 Tourrettes, France \\ ${ }_{5}^{4}$ Editorial Secretary, Solar-Terrestrial Centre of Excellence (STCE), Ringlaan-3-Av. Circulaire, 1180 Brussels, Belgium \\ ${ }^{5}$ Publishing Director, EDP Sciences, 17, av. du Hoggar - BP 112 - 91944 Les Ulis Cdx A, France
}

Received 18 March 2019 / Accepted 18 March 2019

\section{Introduction}

The Journal of Space Weather and Space Climate (indexed either as JSWSC or as SWSC) was created at the end of 2010 to fill a gap in the choice of existing peer-reviewed journals (Lilensten \& Belehaki, 2011). It is an international Gold Open Access journal that publishes papers on all aspects of space weather and space climate from a broad range of scientific and technical fields including solar and heliospheric physics, magnetospheric and ionospheric physics, space plasma physics, aeronomy, planetology, radio science, geophysics, biology, medicine, astronautics, aeronautics, electrical engineering, meteorology, climatology, mathematical physics, economy, and informatics. It was created via a community decision. JSWSC is a community journal which emerged from the COST Action ES0803 "Developing Space Weather Products and Services in Europe" (Belehaki et al., 2014). The Editorial Board was soon expanded to include experts from all over the world. It currently consists of 24 Associate Editors whose expertise covers most aspects of the discipline. These editors have the challenging task to find reviewers, to follow the review process and to make recommendations to the Editors-in-Chief (EiC) about the merit of each article. The Journal of Space Weather and Space Climate is supported by the Editorial Secretary and the Editorial Advisor. It is published by EDP Sciences (http://www.edpsciences.org/) and benefits from strong support from the Solar-Terrestrial Centre of Excellence (STCE) in Brussels, Belgium (http:// www.stce.be). JSWSC publishes only an online electronic version (https://www.swsc-journal.org/) where different formats (HTML, PDF, ePUB) are offered.

In this editorial we present the current status of the journal regarding the flow of submissions, the impact of regular versus topical papers and the length of the publication process. We analyse the review procedure and discuss ways to encourage experts to accept the reviewer invitation. Finally, we present the new Agora Supplement which is intended to provide a forum for articles of high relevance and interest to the space weather and space climate communities but which are not research or technical articles in the strict sense described in the JSWSC aims and scope (https://www.swsc-journal.org/ about-the-journal/aims-and-scope).

\section{Publication activity in the JSWSC}

In this section, we give a brief overview of the journal publication record after eight years of activity. Unless otherwise noted, the Table, Figures and numbers quoted in this paper reflect the status of JSWSC as of 23 October 2018.

Figure 1 shows the number of active manuscripts per week, recorded since the journal's kick-off. An active manuscript is a manuscript that is between the stages of submission and online publication, rejection, withdrawal, or closure.

Starting in May 2017, the number of active manuscripts increased from about 20 to 69 in September 2017, the highest

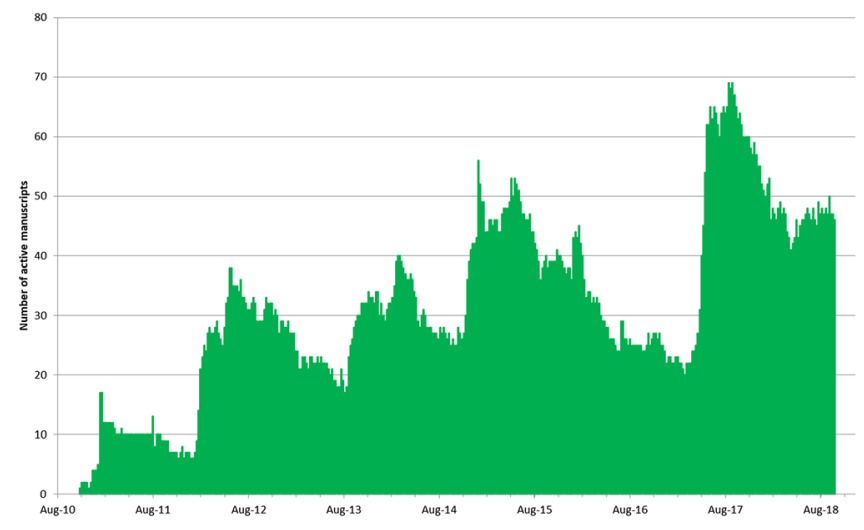

Fig. 1. Number of active manuscripts.

*Corresponding author: jean.lilensten@univ-grenoble-alpes.fr 
since the start of the JSWSC. It has been above 60 from mid-June to mid November 2017. The remarkable increase was due to the opening of four new Topical Issues (TIs). Since April 2018, the number of active manuscripts has stabilized around 45.

A total of 528 papers were submitted, of which 256 were published until October 2018, not including the three Editorials, five Prefaces to Topical Issues and one Erratum.

Overall, there is a positive trend in the number of submissions. The number of submitted manuscripts shown in Figure 1 is heavily driven by the number of Topical Issues. Two Topical Issues were started in 2015, four in 2017. This explains the strong increase seen from mid-2017 onwards. Interestingly, the number of regular papers, i.e., those not belonging to a TI, which remained relatively stable around 20-25 since late 2014, seems to show a slight increase in 2018 (50 for the entire year, compared to 47 and 36 in the two previous years). Figure 2 shows the submissions to the journal, split between regular papers and TIs.

The TIs have an important role in the dissemination of scientific results to the JSWSC related communities around a topic of great current interest. So far, JSWSC has published or is currently publishing 15 TIs. The topics are listed in Table 1 together with the names of the Topical Editors-in-Chief and the number of papers published per TI

The rejection rate in the TIs is $\sim 24 \%$. This is quite low, and is not representative of the journal as a whole. Since the start of the journal, the yearly rejection rate has varied between

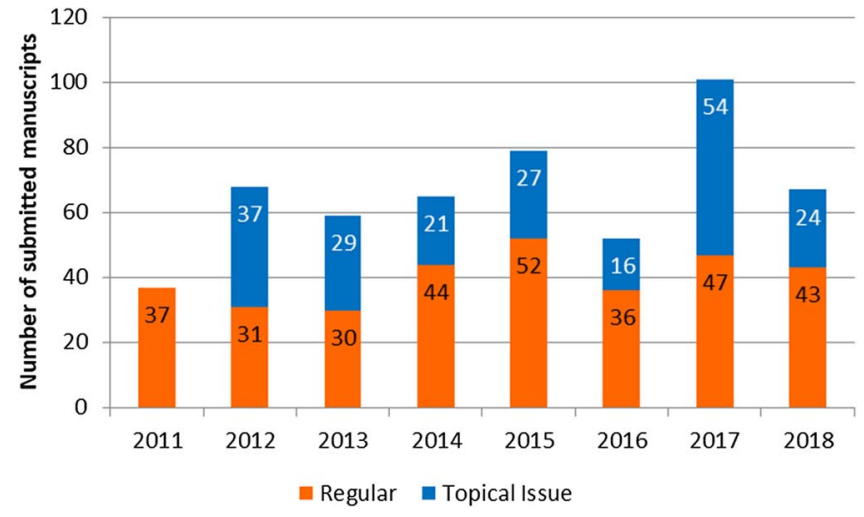

Fig. 2. Submissions to the Journal of Space weather and Space Climate.

$35 \%$ and $45 \%$ with a small decrease in 2017 partly due to the relatively high number of TI papers. This is illustrated in Figure 3.

\section{Review process}

The JSWSC review process consists of several steps, and a manuscript may be rejected at any of them. A scientific article is accepted for publication only after it has successfully passed

Table 1. Overview of all Topical Issues.

Planetary Space Weather (still open)

System Science: Application to Space Weather Analysis, Modelling, and Forecasting (still open)

Developing New Space Weather Tools: Transitioning fundamental science to operational prediction systems

Flares, coronal mass ejections and solar energetic particles and their space weather impacts

Space weather effects on GNSS and their mitigation

Measurement, Specification and Forecasting of the Solar Energetic Particle Environment and GLEs

Brightness Variations of the Sun and Sun-like Stars and Resulting Influences on their Environments

Scientific Challenges in Thermosphere-Ionosphere Forecasting

Statistical Challenges in Solar Information Processing

Satellite mission concepts developed at the Alpbach 2013 Summer School on space weather

Solar variability, solar forcing, and coupling mechanisms in the terrestrial atmosphere

Space Weather and Challenges for Modern Society

COST Action ES0803

EU-FP7 funded space weather projects

Space Climate

$\begin{array}{lr}\text { Nicolas André } & 4 \\ \text { Richard Boynton } & 3 \\ \text { Shaun Bloomfield, Giovanni Lapenta } & 16 \\ \text { Nicole Vilmer, } & 10 \\ \text { Olga Malandraki } & 9 \\ \text { Jens Berdermann, Claudia Borries } & 9 \\ \text { Piers Jiggens, Alexander Mishev } & 10 \\ & \\ \text { Natalie Krivova } & 9 \\ & 7 \\ \text { Anthony J. Mannucci } & 18 \\ \text { Thierry Dudok de Wit } & 6 \\ \text { Periasamy K. Manoharan } & 8 \\ & \\ \text { Thierry Dudok de Wit } & 8 \\ \text { Peter Wintoft } & 12 \\ \text { Maurizio Candidi } & 9 \\ \text { Paola Chiarini } & 13 \\ \text { Kalevi Mursula } & \end{array}$

Left column: title of the issue; middle column: Topical Editor(s)-in-Chief; right column: number of papers published. The most recent TIs (the two top entries) are not closed yet, therefore the low number of published papers. The status date is 1 February 2019 and the numbers include Prefaces and Errata. 


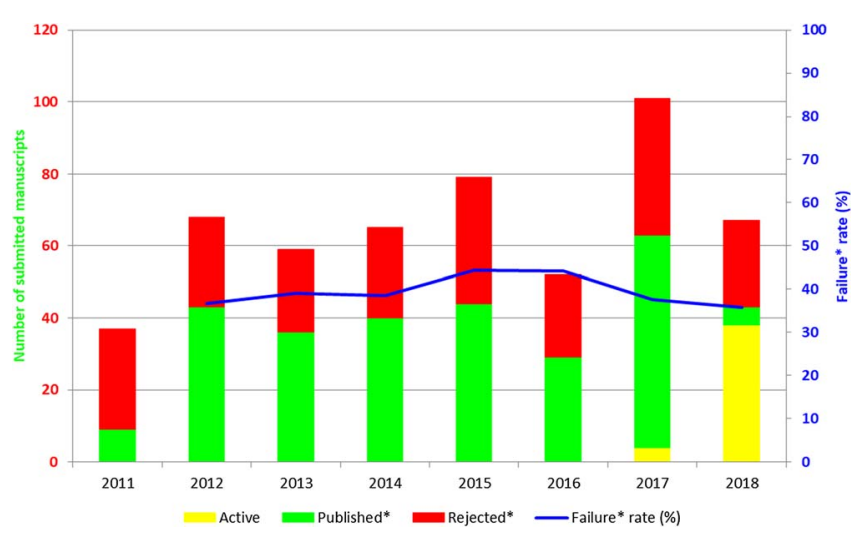

Fig. 3. Evolution of the failure rate per year. Rejected* includes withdrawn and closed manuscripts. Published* includes the editorials and errata. Failure* rate for a year $=$ (Rejected + Withdrawn + Closed)/ALL (including Active).

a peer review process. The first step is the "prescreening" where the two Editors-in-Chief and the Editorial Advisor read the paper, evaluate it independently and discuss its fate. In case they disagree, or in case they do not feel sufficiently competent, they ask one of the Associate Editors (AEs) more specialized in the field for a complementary advice. If the manuscript is recommended for a peer review process, it is transferred to an expert $\mathrm{AE}$ who will handle the review process autonomously. The handling AE may recommend rejection or a revision prior to starting the peer review process. This triggers a discussion between EiCs, Editorial Advisor and handling $\mathrm{AE}$ and results in concluding whether or not to follow the AE recommendation. In case of a Topical Issue, the Topical Editor-in-Chief participates in the discussion. If it is decided that the peer review process should start, the handling $\mathrm{AE}$ has to select and invite referees to review the manuscript and is responsible for getting at least two independent reviews. JSWSC editors are excluded from serving as referees for scientific articles submitted for publication in JSWSC. At the end of the peer review process, which may involve several revisions, the handling $\mathrm{AE}$ makes a recommendation for final decision to the EiCs. In the vast majority of cases, the AE recommendation is followed by the EiCs. The editorial process is not a simple straightforward sequence, it involves a considerable amount of discussion among the editors and an endeavor for consensus.

This multistep procedure partially explains the relatively long JSWSC review process. A regular manuscript requires about 210 days (median) from submission to online publication, with 255 days (median) for a manuscript belonging to a TI. These data concern the 256 published papers, including editorials (3), prefaces (5) and errata (1). The analysis shows that the review process itself is the main driver for such a long duration since the prescreening, while post-review discussions take only about $10 \%$ of the total handling time. The exceptionally long handling times for some manuscripts, sometimes exceeding two years duration (only a few cases), is due to the large number of revisions, to the difficulty in finding available reviewers and to the failure of the authors to respond to the requests for revisions within the allocated time frame. JSWSC allows typically one month for minor revision and two month for major revision.

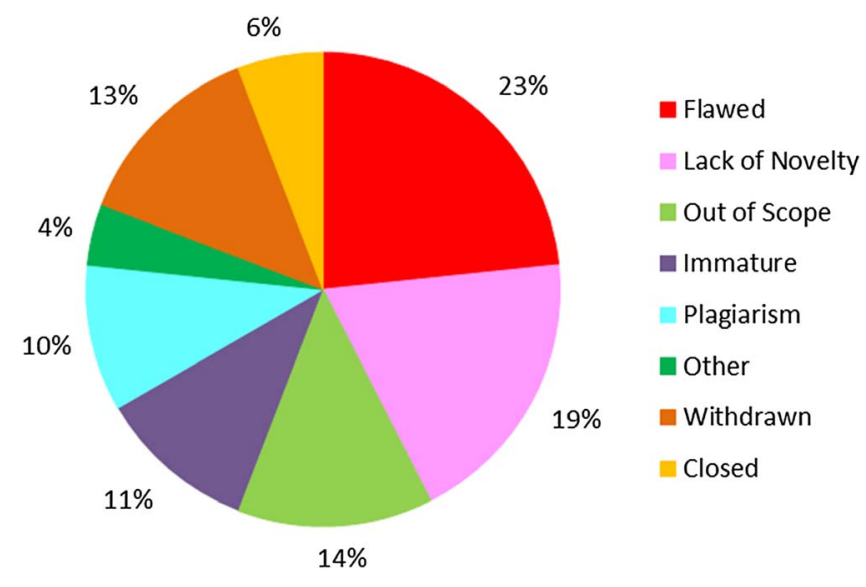

Fig. 4. Reasons for not publishing a manuscript (period 2015-2018).

Rejected papers take less time to reach the final verdict, on average 59 days in the regular issue, and 87 days in the TIs.

The availability of experts who accept our invitations for manuscript reviewing is becoming one of the key issues that often drives the delays in handling new submissions. In some cases more than 10 experts were invited in order to reach the required number of two reviews. It is obvious that we need convincing arguments to bring more and more experts into the review process.

Peer review forms the cornerstone of trusted research outcome validation. The peer review process for scientific journals relies on the efforts of volunteer reviewers. At first sight, with so many demands on professional work hours, the benefits of participating in peer review may not seem obvious. However, reviewers benefit by exposure to the latest developments in their fields, facilitating their keeping up-to-date with the latest publications. Also, it should be considered as a lifelong learning process. The reviewer has to understand deeply the work considered for publication, compare it with the state of the art, and provide constructive comments that will help the authors to improve their work. The reviewer has to provide solid arguments and should be in the position to defend his or her view objectively, in case of dispute with the authors. Moreover, we could point out that contacts made during the peer review process can lead to long-lasting collaborations

Figure 4 shows the percentages of the primary reasons for not publishing a manuscript. EiCs reject directly manuscripts convicted of plagiarism or out of the scope of the journal. In the latter case, we try to suggest more suitable journals to the authors. Some articles are directly rejected because their phrasing is such that it is impossible to figure out what the authors mean. Finally, a small number is rejected for lack of novelty or obvious flaws. Rejections following $A E$ recommendation are usually for lack of novelty or because of flaws in the paper. Rejection for "immaturity" is usually accompanied by an invitation to submit the work as a new, entirely reworked manuscript at some point in time. Manuscripts are closed after about 6 months if no response from the authors is received, despite numerous reminders by both the Editorial Office and the EiCs. 


\section{The JSWSC Agora supplement}

The decision to create a supplement to the JSWSC came from our difficulties to evaluate some articles. All Education and Public Outreach papers and papers on historical observations resulted in tense discussions within the Editorial Board, with final decisions always frustrating for some of the editors. We realised that we were unable to find the proper reviewers. When the manuscripts were reviewed by professionals of communication, the result was often a rejection because the authors - always scientists - never used the communication science criteria. On the other hand, the reviewer was not aware of the constraints due to space weather activities and used irrelevant criteria. In consequence, papers in a new supplement should not be subject to the same review process as in the regular issue of JSWSC.

The creation of this supplement was discussed with the Associate Editors of JSWSC. These discussions resulted in the specification of the characteristics of the supplement which would accommodate publications fundamentally different from our regular research, technical, and review papers. It would not merely be an additional set of new categories but articles of different character:

- reports on Education and Public Outreach activities,

- reports on historical events, inventions, observations, physical concepts,

- commentaries which outline ideas, research concepts, or action plans (e.g., roadmaps) and leave room for expressing personal opinions,

- meeting and project reports.

The Editorial Board at its meeting at the 2017 European Space Weather Week agreed to the launch of the supplement. The articles for the supplement will be internally reviewed by at least four editors, among them the EiCs and the Editorial Advisor.

In order to meet the requests by potential contributors, the new articles in the supplement will be indexed similar to regular JSWSC papers, will receive a DOI and will be citable. However, the supplement will clearly be marked as not peer reviewed.

The Editorial Board approved these recommendations. It agreed to name the new supplement "Agora", a term which denoted in ancient Greek an assembly place for various aspects of public life including intellectual exchange and debate. Our publisher, EDPS, agreed on the principles and will adapt the JSWSC web site to accommodating Agora articles while ensuring a clear distinction from the regular JSWSC articles.

\section{Considerations on the future}

JSWSC is inherently multi-disciplinary and serves a community with a broad range of interests and expertise. To serve this community we benefit from a pool of Associate Editors who possess expertise in one or several of the research fields relevant to JSWSC. So far 79 editors (regular Associate Editors and Guest Editors of Topical Issues) have handled 528 manuscripts. The reviewers also play a key role in this process. Based on 105 published or still active papers submitted in 2017-2018, no less than 195 referees from 36 countries were involved in the review process. A large majority $(84 \%)$ comes from Europe and USA/Canada, and about half come from a country that has a regional space weather warning center (ISES - 13/16 countries represented).

The Journal of Space Weather and Space Climate, with a 2017 two-year Impact Factor of 2.333 and a 2017 five-year Impact Factor of 3.101, is now established and recognized by the space weather and space climate communities. We receive submissions from many different countries all over the world. At the end of 2017, 1,033,916 papers had been downloaded (according to Clarivate Analytics).

These data show that the journal is part of the scientific community and has to grow together with the relevant communities in Europe and worldwide. To meet this challenge, we strive to develop the required tools to facilitate interdisciplinary publications which are representative of all different activities implemented in the scientific fields of space weather and space climate, to meet the requirements of our readers for fast and high-quality publications, and to keep channels open with our readers, followers and supporters in order to establish a sustainable framework of scientific dissemination.

To cope with the growth of JSWSC, we will implement a new manuscript management system which is better adapted to the management of peer reviewed journals with a world-wide author-, referee- and readership than the present one. The change will be felt by authors, reviewers and editors but not by the readers of articles published on the JSWSC web site. This system, called Editorial Manager ${ }^{\circledR}$, is already used by thousands of journals across many fields of research and therefore probably not unfamiliar to authors and reviewers. We expect that its special features will help to reduce the time delay between submission of a manuscript and the final decision and online publication. We work for a smooth transition to the new system, but we would like to apologize in advance for problems which may turn up and errors which may occur in the initial phase of our using the new system.

In order to maintain a wide reader interest, it is important to launch new Topical Issues focused on topics that are of high relevance and impact. It is equally important to attract the interest of the relevant communities with innovations such as the new Agora supplement.

In 2018, we organised an open session at the European Space Weather Week in Leuven to discuss our strategy and get a feedback from the related communities. Ideas to reduce the publication time have been discussed. Among others, this concerns actions of encouraging colleagues to serve as reviewers. A new international initiative merits to be mentioned. Publons (https://publons.com/about/home/) is a new way to track the publications, citation metrics, peer reviews, and journal editing work in a single profile. Not only the publications and citation metrics are imported from Web of Science, but the verified peer review and journal editing history are listed. The Journal of Space Weather and Space Climate encourages its reviewers and editors to mention their contributions to the Journal through this venue.

Last not least we are grateful to the authors, reviewers, Associate Editors, and our publisher EDPS for their confidence they have put in JSWSC and their active contribution to its success. 


\section{References}

Belehaki A, Messerotti M, Candidi M. 2014. Developing space weather products and services in Europe - Preface to the Special
Issue on COST Action ES0803. J Space Weather Space Clim 4: E1. DOI: $10.1051 / \mathrm{swsc} / 2014032$.

Lilensten J, Belehaki A. 2011. Editorship at SWSC. J Space Weather Space Clim 1: E01. DOI: 10.1051/swsc/2011002.

Cite this article as: Lilensten J, Belehaki A, Watermann J, Janssens J \& Henri A. 2019. JSWSC: recent developments and further advances. J. Space Weather Space Clim. 9, E2. 